\title{
Desempenho produtivo e características de carcaça de cordeiros Rabo Largo puro e cruzados com Santa Inês
}

[Performance and carcass traits of Rabo Largo lambs and crossbreeding with Santa Inês]

\author{
A.V. Landim ${ }^{1,6}$, H.H.A. Costa ${ }^{1, *}$, F.C. Carvalho ${ }^{1}$, A.C. Costa ${ }^{3}$, R.T. Alencar ${ }^{2}$, L.N.C. Silva ${ }^{2}$, J.S. Gomes ${ }^{3}$, \\ A.S.M. Batista ${ }^{1}$, E.S. Miyagi ${ }^{4}$, L.D. Lima $^{5}$ \\ ${ }^{1}$ Universidade Estadual Vale do Acaraú - UVA - Sobral, CE \\ ${ }^{2}$ Aluno do Programa de Pós-Graduação em Zootecnia da Universidade Estadual Vale do Acaraú - UVA - Sobral, CE \\ ${ }^{3}$ Aluno do Curso de Graduação em Zootecnia da Universidade Estadual Vale do Acaraú - UVA - Sobral, CE \\ ${ }^{4}$ Escola de Medicina Veterinária e Zootecnia - Universidade Federal de Goiás - UFG- Goiânia, GO \\ ${ }^{5}$ Embrapa Caprinos e Ovinos - Sobral, CE \\ ${ }^{6}$ Bolsista de Produtividade em Pesquisa, Estímulo à Interiorização e à Inovação \\ Tecnológica - BPI/FUNCAP (Edital 09/2015). No do Processo: BP2-0107-00040.01.00/15
}

\section{RESUMO}

Objetivou-se avaliar o efeito do cruzamento entre ovinos nativos no desempenho e características da carcaça. Foram utilizados vinte cordeiros, machos, inteiros, com idade inicial de quatro meses, e peso médio inicial 19,0 $\pm 5,11$, distribuídos em dois grupos genéticos: Rabo Largo (RL) e Santa Inês x Rabo Largo ( $F_{1} S I x$ RL), em um delineamento inteiramente ao acaso. Os animais foram terminados em regime de confinamento durante 70 dias. Os cordeiros $\mathrm{F}_{1} \mathrm{SI}$ x RL apresentaram maiores pesos ao desmame, ao abate, do corpo vazio, das carcaças quente e fria, e maior rendimento biológico. Verificaram maiores valores para os parâmetros de morfometria da carcaça para animais $\mathrm{F}_{1} \mathrm{SI} x \mathrm{RL}$ comparados aos animais Rabo Largo $(\mathrm{P}<0,05)$. Os pesos da hemi-carcaça e dos cortes comerciais foram superiores para cordeiros $\mathrm{F}_{1} \mathrm{SI}$ x RL. Nas medidas obtidas no músculo Longissimus dorsi, os cordeiros Rabo Largo apresentaram valores superiores para espessura de gordura subcutânea. A utilização da raça Santa Inês como base paterna acarreta em melhorias nas características de carcaça de cordeiros Rabo Largo podendo ser indicado em sistemas de produção de carne ovina.

Palavras-chave: ovinos, cruzamento, naturalizados, rendimento de carcaça

\begin{abstract}
The aim of study was to evaluate the effect of crossbreeding among native sheep on performance and traits carcass. Twenty intact lambs, male, at four months of age, with an initial weight of $19.0 \pm 5.11 \mathrm{~kg}$, were distributed in two groups genetics: Rabo Largo (RL) e Santa Inês x Rabo Largo (F1SI x RL), in a completely randomized design. The animals were finished in feedlot by 70 days. The lambs $F_{1} S I \times$ RL showed higher weight at weaning, to slaughter, of the empty body, and hot and cold carcass, and increased biological yield. Higher values for carcass morphometry in $F_{1} S I \times R L$ animals were found in comparison to Rabo Largo animals $(P<0.05)$. The weight of half-carcass and the commercial cuts were higher for lambs. On the measurements obtained in muscle Longissimus dorsi, Rabo Largo lambs showed higher values for subcutaneous fat thickness. The use of the breed Santa Inês like base paternal may bring in improvements in lambs's carcass characteristics Rabo Largo and may be recommended in sheep meat production systems.
\end{abstract}

Keywords: sheep, carcass yield, crossbreeding, naturalized

\section{INTRODUÇÃO}

$\mathrm{Na}$ região Nordeste do Brasil a maioria dos rebanhos ovinos são constituídos por raças localmente adaptadas, resistentes às condições edafoclimáticas, contudo, são raças que carecem

Recebido em 31 de outubro de 2016

Aceito em 4 de janeiro de 2017

*Autor para correspondência (corresponding author)

E-mail: helioa.costa@gmail.com de precocidade de acabamento e qualidade de carcaça (Barros et al., 2005). A eficiência na produção de carne ovina na região Nordeste, assim como nos demais centros de produção ovina, pode ser aprimorada utilizando-se sistemas de cruzamento planejados, aliado a manejos produtivos adequados (Carneiro et al., 2007). 
Os animais oriundos de cruzamentos apresentam potencial em reduzir a idade ao abate e além dos custos de produção, podendo acarretar em melhorarias na produção de carcaças com características desejadas em termos de maior deposição de músculos e acabamento, implicando em produtos com potencial de aceitação e comercialização no seguimento do mercado da carne (Madruga et al., 2006).

Os ovinos Rabo Largo destacam-se entre as raças naturalizadas exploradas, principalmente no Nordeste, tendo como característica marcante a deposição de gordura na cauda (McManus et al., 2010). No entanto, embora com poucos exemplares da raça, que estão localizados em pequenos núcleos, e em alguns estados como Bahia, Paraíba e Ceará, apresentam potencial para uso nos sistemas de produção ovina em decorrência de sua rusticidade (Carvalho, 2013). Por outro lado, a raça Santa Inês é caracterizada pelo grande porte e potencial para produção de carne (Furusho-Garcia et al., 2010) sendo a de maior efetivo na região Nordeste, além de constituir boa parte dos rebanhos de outras regiões como o Sudeste e Centro-Oeste do Brasil.

Os estudos relacionados às características quantitativas e qualitativas de carcaça apresentam-se como ferramenta importante na identificação de produtos de qualidade, sendo necessária a avaliação dos animais com intuito de caracterizar as diferenças existentes entre os genótipos utilizados e seus cruzamentos (Cartaxo et al., 2011).

Acredita-se que animais localmente adaptados em sistemas de cruzamento pode ser uma alternativa no processo de conservação destes recursos genéticos animais, além de contribuir para elevar o efetivo destes animais nos rebanhos. Dessa forma, objetivou-se avaliar o desempenho e as características da carcaça de cordeiros Rabo Largo e Santa Inês x Rabo Largo.

\section{MATERIAL E MÉTODOS}

Todos os procedimentos e manuseio dos animais experimentais foram realizados de acordo com os protocolos aprovados pelo Comitê de Ética no Uso de Animais da Universidade Estadual Vale do Acaraú - UVA (protocolo $\mathrm{n}^{\circ}$ 003.04.015.UVA.504.02).
O experimento foi conduzido na Fazenda Experimental da Universidade Estadual Vale do Acaraú, Sobral, CE. Foram utilizados vinte cordeiros, machos, não castrados, peso médio de $19,0 \pm 5,11 \mathrm{~kg}$, e quatro meses de idade, alocados em baias individuais. Para composição dos tratamentos experimentais, foram considerados os grupos genéticos Rabo Largo (RL) e Santa Inês x Rabo Largo ( $F_{1} S I$ x RL).

Os animais foram alojados em baias individuais com área de $1,2 \mathrm{~m}^{2}$, providas de comedouros, bebedouros e saleiros e terminados em regime de confinamento durante 70 dias, com 14 dias para adaptação e 56 dias para avaliação do desempenho. Os animas foram alimentados com dieta constituída de capim Canarana erecta lisa (Echinocloa pyramidalis) e ração concentrada à base de milho, farelo de soja e calcário numa relação 60:40 volumoso:concentrado formulada conforme recomendações do NRC (Nutrient..., 2007), para categoria de terminação e previsão de ganho de peso médio de $150 \mathrm{~g} \mathrm{dia}^{-1}$.

A dieta foi fornecida em duas refeições iguais (às $8 \mathrm{~h}$ e às 16h) permitindo-se sobras diárias de aproximadamente $10 \%$ do total fornecido para proporcionar ingestão voluntária. Água e sal mineralizado foram disponibilizados à vontade. Os animais foram pesados semanalmente para estimativa do ganho de peso total e ganho de peso médio diário $(\mathrm{GPMD}=$ ganho de peso total/período experimental).

Foi realizado o acompanhamento do desempenho ponderal dos ovinos, para obtenção de ganho de peso médio diário (GPMD, g) e ganho total (GPT, kg).

Os componentes da dieta (capim e concentrado) foram analisados para matéria seca (MS, método, 934.01), cinzas (método 938.08) extrato etéreo (EE, método 920.39) e proteína bruta (PB, método 934.13) conforme recomendação da AOAC (Official..., 1990). A matéria orgânica (MO) foi calculada como a diferença entre o teor de MS e cinzas. Fibra em detergente neutro (FDN), fibra em detergente ácido (FDA) foram analisados de acordo com Van Soest et al. (1991). Lignina em detergente ácido foi determinada segundo o método 973.18D (Official..., 1990). Para análise da digestibilidade in vitro da MS e MO utilizou-se a metodologia de Tilley e Terry (1963) (Tab. 1). 
Tabela 1. Composição dos componentes dietéticos

\begin{tabular}{|c|c|c|c|}
\hline \multirow{2}{*}{ Nutrientes $(\%)$} & \multicolumn{3}{|c|}{ Componentes da dieta } \\
\hline & Capim $^{*}$ & Concentrado $^{\beta}$ & Dieta \\
\hline Matéria seca (MS) & 91,8 & 92,9 & 92,2 \\
\hline Matéria orgânica (MO) & 87,2 & 94,5 & 90,1 \\
\hline Proteína bruta & 5,16 & 36,8 & 17,8 \\
\hline Extrato etéreo & 1,28 & 4,00 & 2,37 \\
\hline Fibra em detergente neutro & 75,8 & 16,9 & 52,2 \\
\hline Fibra em detergente ácido & 43,0 & 9,60 & 29,6 \\
\hline Hemicelulose & 32,5 & 7,30 & 22,4 \\
\hline Celulose & 34,7 & 9,43 & 24,6 \\
\hline Lignina & 4,61 & 0,37 & 2,91 \\
\hline Digestibilidade in vitro MS & 31,0 & 73,0 & 47,8 \\
\hline Digestibilidade in vitro $\mathrm{MO}$ & 38,7 & 81,3 & 55,7 \\
\hline
\end{tabular}

Os animais foram abatidos com aproximadamente $26,0 \pm 2,13 \mathrm{~kg}$ de peso vivo médio. Previamente aos procedimentos de abate, os animais foram submetidos a jejum de dieta sólida por 16 horas. Após o jejum, foram pesados e obtido o peso vivo ao abate (PVA). O abate foi realizado de acordo com as normas de regulamentação de inspeção industrial e sanitária de produtos de origem animal (Brasil, 1997). Após a evisceração, foram pesados os constituintes não carcaça. O peso do corpo vazio (PCVZ) conforme Silva Sobrinho (2001). A carcaça quente foi pesada e acondicionada em câmara fria, à temperatura de $4^{\circ} \mathrm{C}$, por 24 horas. Posteriormente, a carcaça fria foi pesada. A partir dos pesos da carcaça quente (PCQ) e fria (PCF) foram calculados parâmetros relacionados às carcaças como: rendimento carcaça quente $(\mathrm{RCQ})=(\mathrm{PCQ} / \mathrm{PVA}) \times 100$; rendimento carcaça fria $(\mathrm{RCF})=(\mathrm{PCF} / \mathrm{PCQ}) \times 100$; perda por resfriamento $(\mathrm{PR})=[(\mathrm{PCQ}-\mathrm{PCF}) / \mathrm{PCQ}] \times 100$, e rendimento biológico verdadeiro $(\mathrm{RBV})=$ (PCQ/PCVZ) x 100.

Foram realizadas mensurações de morfometria e conformação da carcaça (Osório et al., 1998; Cezar e Sousa, 2007). Em seguida, foi seccionada longitudinalmente e realizado os cortes comerciais, pesados, e determinado os rendimentos em relação ao peso da hemi-carcaça reconstituída (Silva Sobrinho, 2001).

No músculo Longissimus dorsi, na altura da $12^{\mathrm{a}}$ vértebra torácica, foi calculado a área de olho de lombo pelo padrão gabarito transparente quadriculado, $1 \mathrm{~mm}^{2}$ célula $^{-1}$ (Cunha et al., 2001), e com uso de paquímetro digital mensurado a espessura de gordura subcutânea (EGS) e as avaliações de textura, marmoreio, distribuição do marmoreio e cor de acordo com a metodologia descrita por Osório et al., (2002).

O delineamento utilizado foi o inteiramente ao acaso, dois tratamentos e dez repetições, utilizando-se o seguinte modelo estatístico:

$\mathrm{Y}_{\mathrm{ijk}}=\mu+\mathrm{GG}_{\mathrm{i}}+\mathrm{e}_{\mathrm{ij}}$;

Em que, $\mu=$ média geral;

$\mathrm{GG}_{\mathrm{i}}=$ efeito dos grupos genéticos $\left(\mathrm{i}=\mathrm{RL}\right.$ e $\mathrm{F}_{1} \mathrm{SI}$

$x$ RL) e

$\mathrm{e}_{\mathrm{ijk}}=$ erro aleatório.

Utilizou-se o procedimento Proc GLM do Statistical Analysis System - SAS ${ }^{\circledR} 9.0$ (2001). As médias foram comparadas utilizando-se o teste Tukey, admitindo-se significância de $\mathrm{P}>0,05$.

\section{RESULTADOS E DISCUSSÃO}

Para peso ao nascer houve similaridade entre cordeiros puros e cruzados com valores médios de 2,80 e $3,13 \mathrm{~kg}$, respectivamente $(\mathrm{P}>0,05$; Tab. 2).

A utilização da raça Santa Inês como base paterna propiciou aos cordeiros cruzados maior peso ao desmame, sendo observado $33 \%$ de superioridade em relação aos animais Rabo Largo puro, assim como, a sua participação crescente no desempenho destes animais, devido 
ao efeito da heterose, que proporcionou melhorias no desempenho de animais cruzados, sendo estes considerados especializados para a produção de carne.

Para ganho de peso médio diário (GPMD) e ganho de peso total (GPT) não houve diferenças entre os grupos genéticos $(\mathrm{P}>0,05$; Tab. 2). A paridade nestes parâmetros entre os cordeiros puros e cruzados indicaram que animais jovens independentes do tipo racial, se mantidos em condições adequadas de nutrição e sanidade apresentam condição para ganhos em peso de forma similar.

Tabela 2. Efeito do cruzamento em cordeiros Rabo Largo e Santa Inês x Rabo Largo no desempenho, pesos e rendimentos de carcaça

\begin{tabular}{|c|c|c|c|}
\hline \multirow{2}{*}{ Variáveis } & \multicolumn{2}{|c|}{ Grupos Genéticos } & \multirow{2}{*}{$\mathrm{EPM}^{¥}$} \\
\hline & $\mathrm{F}_{1} \mathrm{SI} \times \mathrm{RL}^{€}$ & $\mathrm{RL}^{\mathfrak{f}}$ & \\
\hline \multicolumn{4}{|c|}{ Desempenho e parâmetros de carcaça, $\mathrm{kg}$} \\
\hline Peso ao nascer & 3,13 & 2,80 & 0,166 \\
\hline Peso ao desmame & $22,8^{\mathrm{a}}$ & $15,1^{\mathrm{b}}$ & 1,111 \\
\hline Ganho de peso médio diário & 0,060 & 0,045 & 0,205 \\
\hline Ganho de peso total & 4,17 & 5,95 & 0,952 \\
\hline Peso ao abate & $28,6^{\mathrm{a}}$ & $24,1^{b}$ & 0,960 \\
\hline Peso do corpo vazio & $20,1^{\mathrm{a}}$ & $16,9^{\mathrm{b}}$ & 0,765 \\
\hline Peso da carcaça quente & $11,9^{\mathrm{a}}$ & $9,36^{\mathrm{b}}$ & 0,573 \\
\hline Peso da carcaça fria & $11,3^{\mathrm{a}}$ & $8,69^{b}$ & 0,569 \\
\hline \multicolumn{4}{|l|}{ Parâmetros de carcaça, \% } \\
\hline Perda de peso por resfriamento & 5,04 & 7,15 & 0,576 \\
\hline Rendimento da carcaça quente & 41,4 & 38,5 & 0,834 \\
\hline Rendimento da carcaça fria & 38,9 & 35,7 & 0,877 \\
\hline Rendimento biológico verdadeiro & $59,1^{\mathrm{a}}$ & $55,0^{\mathrm{b}}$ & 1,020 \\
\hline
\end{tabular}

${ }^{\epsilon} \mathrm{F}_{1}$ SI x RL: Santa Inês x Rabo Largo; ${ }^{\mathfrak{E}} \mathrm{RL}$ : Rabo Largo; ${ }^{*} \mathrm{EPM}$ = Erro-padrão da média. Valores na mesma linha, seguidos de letras diferentes, diferem estatisticamente $(\mathrm{P}<0,05)$ pelo teste Tukey $(\mathrm{P}<0,05)$.

Verificou-se que apesar dos animais terem apresentado ganho de peso inferior, os resultados foram próximos aos obtidos por Furusho-Garcia et al. (2010) que obtiveram ganho de peso médio de $84 \mathrm{~g}$ em cordeiros Santa Inês e seus cruzamentos com raças Dorper e Texel terminados em sistema extensivo com capim aruana (Panicum maximum cv Aruana). Já Costa et al. (2011) verificaram em cordeiros Santa Inês, $\mathrm{F}_{1}$ Dorper $\mathrm{x}$ Santa Inês e sem padrão racial definido (SPRD) alimentados com dietas isoproteicas ganhos de peso de 240,6; 223,0 e $229,9 \mathrm{~g}$, respectivamente.

Houve diferença entre os grupos genéticos para os parâmetros de peso vivo ao abate (PVA) e do corpo vazio (PCVZ) (Tab. 2; $\mathrm{P}<0,05)$. Os cordeiros RL apresentaram menores pesos em relação ao grupo $\mathrm{F}_{1} \mathrm{SI} \times \mathrm{RL}$, o que era esperado, uma vez que são animais não especializados para corte, quando comparado com os cruzas Santa Inês.
Os pesos de carcaça quente (PCQ) e fria (PCF) diferiram entre os grupos genéticos $(\mathrm{P}<0,05)$ com maiores pesos para cordeiros $\mathrm{F}_{1} \mathrm{SI} \times \mathrm{RL}$ decorrente do maior peso ao abate, uma vez que os pesos de carcaça apresentam alta correlação com o peso de abate (Piola Junior et al., 2009).

Não foram observadas diferenças $(\mathrm{P}>0,05)$ entre os genótipos para rendimentos de carcaça quente (RCQ) e fria (RCF) com valores de 46,0\% e $44,5 \%$ onde ficaram abaixo dos preconizados por Silva Sobrinho (2001) para obtenção de carcaças que apresentem boa qualidade. No entanto, os valores inferiores para os rendimentos podem estar relacionados com o alto percentual de conteúdo gastrintestinal que implicou em 21,2\% do peso vivo ao abate (Tab. 4). De acordo com Siqueira et al. (2001) o conteúdo gastrintestinal é um dos fatores determinantes no rendimento da carcaça.

$\mathrm{O}$ rendimento biológico foi afetado pelo grupo genético ( $\mathrm{P}<0,05$; Tab. 2), contudo, os maiores 
valores para os cordeiros $\mathrm{F}_{1} \mathrm{SI} \quad \mathrm{x} \quad \mathrm{RL}$ apresentaram média $59,1 \%$, sendo $7,45 \%$ maior que o rendimento dos cordeiros RL. Não houve efeito $(\mathrm{P}>0,05)$ para a perda de peso por resfriamento, contudo, foram verificados valores superiores quando comparado aos encontrados na literatura.

A maior perda de peso por resfriamento observada na carcaça dos cordeiros de 6,09\%, pode estar associada a menor espessura de gordura subcutânea encontrada nos cordeiros $\mathrm{F}_{1} \mathrm{SI} x$ RL e RB de 1,24 e 2,41mm, respectivamente (Tab. 3), responsável pela proteção da carcaça no momento do resfriamento. Silva Sobrinho (2001) preconiza que a espessura de gordura subcutânea deve apresentar valores entre 3 e $5 \mathrm{~mm}$, uma vez que valores inferiores podem ocasionar aumento de perdas de peso por resfriamento, encurtamento das fibras musculares em decorrência do frio e escurecimento da parte externa dos músculos. Em pesquisa com cordeiros $F_{1}$ Santa Inês x SPRD e $\mathrm{F}_{1}$ Somalis Brasileiro $\mathrm{x}$ SPRD foram verificados valores de 3,60 e 3,80\% para peso de peso por resfriamento (Oliveira et al., 2014). De forma geral, os índices de perda de peso por resfriamento devem estar em torno de 2,5\%, embora exista oscilação entre 1 e $7 \%$, de acordo com a uniformidade da cobertura de gordura, sexo, peso, temperatura e umidade relativa da câmara de resfriamento (Martins et al., 2000).

A morfometria da carcaça foi afetada pelos cruzamentos $\left(\mathrm{P}<0,05\right.$, Tab. 3). Os cordeiros $\mathrm{F}_{1} \mathrm{SI}$ $\mathrm{X}$ RL apresentaram valores superiores para comprimentos da carcaça e do pernil, e para conformação, já que os animais Santa Inês são caracterizados por ser longilíneo e pernalta (Costa et al., 2014), explicando os maiores valores para os parâmetros da morfometria nos animais cruzados.

Tabela 3. Efeito do cruzamento em cordeiros Rabo Largo e $\mathrm{F}_{1} \mathrm{SI}$ x RL nas características morfométricas da carcaça

\begin{tabular}{|c|c|c|c|}
\hline \multirow{2}{*}{ Variáveis } & \multicolumn{2}{|c|}{ Grupos Genéticos } & \multirow{2}{*}{$\mathrm{EPM}^{\ddagger}$} \\
\hline & $\mathrm{F}_{1} \mathrm{SI}_{\mathrm{R} \mathrm{RL}^{\epsilon}}$ & Rabo Largo $^{£}$ & \\
\hline \multicolumn{4}{|l|}{ Morfometria da carcaça, $\mathrm{cm}$} \\
\hline Comprimento da carcaça & $53,6^{\mathrm{a}}$ & $48,2^{\mathrm{b}}$ & 0,917 \\
\hline Comprimento do pernil & $35,9^{a}$ & $33,9^{\mathrm{b}}$ & 0,424 \\
\hline Comprimento interno da carcaça & 59,6 & 55,6 & 1,130 \\
\hline Largura da garupa & 11,0 & 10,0 & 0,328 \\
\hline Perímetro do pernil & 24,8 & 23,7 & 0,363 \\
\hline Perímetro torácico & 61,6 & 58,6 & 1,010 \\
\hline Acabamento (1-5) & 2,65 & 2,50 & 0,127 \\
\hline Conformação (1-5) & $2,85^{\mathrm{a}}$ & $2,45^{\mathrm{b}}$ & 0,103 \\
\hline Área de olho de lombo $\left(\mathrm{cm}^{2}\right)$ & 8,00 & 7,20 & 0,460 \\
\hline Espessura de gordura (mm) & $1,24^{\mathrm{b}}$ & $2,41^{\mathrm{a}}$ & 0,235 \\
\hline Marmoreio da gordura (1-5) & 1,60 & 1,60 & 0,112 \\
\hline Distribuição do marmoreio da gordura (1-5) & 1,40 & 1,40 & 0,134 \\
\hline Cor $(1-5)$ & $3,10^{\mathrm{a}}$ & $2,40^{\mathrm{b}}$ & 0,160 \\
\hline Textura da carne (1-5) & 4,10 & 4,10 & 0,135 \\
\hline
\end{tabular}

${ }^{\epsilon_{\mathrm{F}}} \mathrm{SI}_{1}$ x RL: Santa Inês x Rabo Largo; ${ }^{\ell} \mathrm{RL}$ : Rabo Largo; ${ }^{*} \mathrm{EPM}=$ Erro-padrão da média. Valores na mesma linha, seguidos de letras diferentes, diferem estatisticamente $(\mathrm{P}<0,05)$ pelo teste Tukey $(\mathrm{P}<0,05)$.

No entanto, o baixo ganho de peso durante o período experimental, resultou em similaridade entre as medidas de largura de garupa e perímetro torácico e de pernil, com valores médios de 10,51; 60,1 e 24,25cm, respectivamente, fato que denota baixo desenvolvimento muscular entre os grupos genéticos. A previsão de ganho de peso médio foi de $150 \mathrm{dia}^{-1}$. No entanto, o ganho não foi alcançado, uma vez que a fração volumosa da dieta apresentou baixa concentração de proteína bruta e da digestibilidade da matéria seca e matéria orgânica (Tab. 1), fato que possivelmente pode ter contribuído para os baixos ganhos de peso.

Ressalta-se que a conformação é uma medida subjetiva utilizada como critério para classificar a carcaça através da avaliação visual para estimar a quantidade de músculo da carcaça (Cezar e 
Sousa, 2007). Houve diferença entre os grupos genéticos para conformação $(\mathrm{P}<0,05$; Tabela 3$)$. Os cordeiros cruzados $\mathrm{F}_{1} \mathrm{SI}$ x RL apresentaram carcaça de melhor conformação da carcaça em relação aos RL, ou seja, melhor distribuição de músculo na carcaça. Amaral et al. (2011) verificaram que os cordeiros cruzados $1 / 2$ DorperSanta Inês e $1 / 2$ White Dorper-Santa Inês apresentaram melhor conformação de carcaça quando comparados com os da raça Santa Inês.

Neste estudo a espessura de gordura subcutânea foi superior nos cordeiros Rabo Largo quando comparados aos cordeiros cruzados, contudo, essa diferença pode ter sido atribuída à maior aptidão dos cordeiros Rabo Largo em depositar tecido adiposo na carcaça. No entanto, a menor espessura de gordura subcutânea verificada nos cordeiros pode estar relacionada à idade reduzida dos cordeiros ao abate, os quais apresentaram média de 180 dias de idade, logo os animais não tiveram tempo necessário para que a camada de gordura subcutânea fosse depositada na carcaça em maior proporção, ou seja em termos de extensão e de espessura.
A cor é uma das primeiras características observadas pelos consumidores no ato da compra, constituindo o critério básico para a sua escolha (Silva et al., 2008). Neste estudo, os cordeiros cruzados $\mathrm{F}_{1} \mathrm{SI}$ x RL apresentaram carne com coloração mais intensa que os cordeiros puros. Dessa forma, uma possível causa da diferença encontrada na cor da carne seja característica da heterogeneidade racial, uma vez que foi aplicado manejo semelhante em ambos os grupos, e foram abatidos na mesma faixa etária. A cor da carne pode variar ainda em função da dieta e idade dos animais (Cezar e Sousa, 2007).

Houve diferença para os componentes nãocarcaça entre os grupos genéticos $(\mathrm{P}<0,05$; Tabela 4). Os cordeiros F $_{1}$ SI x RL apresentaram maiores pesos de sangue, trato gastrointestinal cheio, conteúdo gastrintestinal e pulmão+traquéia em relação ao RL. Contudo, não houve influência no rendimento de carcaça, mas é necessário considerar além do porte, a maturidade dos animais (Martins, 2014), o que possivelmente influenciou nos maiores pesos dos cordeiros $\mathrm{F}_{1} \mathrm{SI} x \mathrm{RL}$.

Tabela 4. Efeito do cruzamento em cordeiros Rabo Largo puro e $\mathrm{F}_{1} \mathrm{SI}$ x RL nos componentes não-carcaça

\begin{tabular}{|c|c|c|c|}
\hline \multirow{2}{*}{ Variáveis } & \multicolumn{2}{|c|}{ Grupos Genéticos } & \multirow{2}{*}{$\mathrm{EPM}^{¥}$} \\
\hline & $\mathrm{F}_{1} \mathrm{SI}_{\mathrm{R} \mathrm{RL}^{€}}$ & Rabo Largo $^{£}$ & \\
\hline \multicolumn{4}{|l|}{$S P C P^{\#}$ e trato digestivo, $\mathrm{kg}$} \\
\hline Sangue & $1,08^{\mathrm{a}}$ & $0,810^{\mathrm{b}}$ & 0,048 \\
\hline Pele & 2,17 & 2,05 & 0,084 \\
\hline Cabeça & 1,63 & 1,87 & 0,052 \\
\hline Patas & 0,565 & 0,480 & 0,023 \\
\hline TGI cheio & $8,540^{\mathrm{a}}$ & $7,170^{\mathrm{b}}$ & 0,220 \\
\hline TGI vazio & 2,430 & 2,070 & 0,093 \\
\hline Conteúdo Gastrintestinal & $6,110^{\mathrm{a}}$ & $5,100^{\mathrm{b}}$ & 0,157 \\
\hline \multicolumn{4}{|l|}{ Órgãos vermelhos, $\mathrm{kg}$} \\
\hline Pulmão + traqueia & $0,545^{\mathrm{a}}$ & $0,455^{\mathrm{b}}$ & 0,019 \\
\hline Coração & 0,097 & 0,108 & 0,012 \\
\hline Rins & 0,081 & 0,072 & 0,002 \\
\hline Fígado & 0,419 & 0,376 & 0,015 \\
\hline Baço & 0,078 & 0,059 & 0,019 \\
\hline Pâncreas & 0,033 & 0,045 & 0,012 \\
\hline
\end{tabular}

${ }^{\epsilon} \mathrm{F}_{1}$ SI x RL: Santa Inês x Rabo Largo; ${ }^{\mathfrak{R}} \mathrm{RL}$ : Rabo Largo; EPM = Erro-padrão da média; TGI = Trato gastrointestinal; ${ }^{a}$ Valores na mesma linha, seguidos de letras diferentes, diferem estatisticamente $(\mathrm{P}<0,05)$ pelo teste Tukey $(\mathrm{P}<0,05)$. ${ }^{\#} \mathrm{SPCP}=$ Sangue, pele, cabeça e pastas.

Para os parâmetros de carcaça não houve efeito do grupo genético para os pesos dos cortes comerciais e seus rendimentos ( $\mathrm{P}>0,05$; Tab. 5), exceto, para os pesos de pernil, paleta e costela e rendimento de costela que foram superiores nos cordeiros cruzados $\mathrm{F}_{1} \mathrm{SI} x \mathrm{RL}$.

Entre os cortes comerciais, tais como pernil, paleta e lombo, por se tratar de cortes nobres, 
apresentam participação significativa e são os mais valorizados da carcaça (Pompeu et al., 2012). Considerando-se que o percentual dos cortes foi de $61,2 \%$, onde o valor apresentado está dentro da faixa preconizado por Silva
Sobrinho et al. (2005) que descreveu que em raças produtoras de carne a soma dos rendimentos dos cortes nobres devem apresentar valor superior a $60 \%$.

Tabela 5. Efeito do cruzamento em cordeiros Rabo Largo e $\mathrm{F}_{1} \mathrm{SI} x \mathrm{RL}$ no peso e rendimento dos cortes comerciais

\begin{tabular}{|c|c|c|c|}
\hline \multirow{2}{*}{ Variáveis } & \multicolumn{2}{|c|}{ Grupos Genéticos } & \multirow{2}{*}{$\mathrm{EPM}^{¥}$} \\
\hline & $\mathrm{F}_{1} \mathrm{SI} \times \mathrm{RL}^{\epsilon}$ & Rabo Largo ${ }^{£}$ & \\
\hline \multicolumn{4}{|l|}{ Pesos, $\mathrm{kg}$} \\
\hline Hemi-carcaça ${ }^{\mathfrak{f}}$ & $5,59^{\mathrm{a}}$ & $4,37^{\mathrm{b}}$ & 0,264 \\
\hline Pernil & $1,78^{\mathrm{a}}$ & $1,46^{\mathrm{b}}$ & 0,079 \\
\hline Paleta & $1,08^{\mathrm{a}}$ & $0,822^{\mathrm{b}}$ & 0,051 \\
\hline Lombo & 0,526 & 0,422 & 0,035 \\
\hline Serrote & 0,823 & 0,709 & 0,037 \\
\hline Costela & $0,922^{\mathrm{a}}$ & $0,561^{\mathrm{b}}$ & 0,058 \\
\hline Pescoço & 0,461 & 0,393 & 0,028 \\
\hline \multicolumn{4}{|l|}{ Rendimentos, \% } \\
\hline Pernil & 31,9 & 33,4 & 0,373 \\
\hline Paleta & 19,3 & 18,9 & 0,348 \\
\hline Lombo & 9,26 & 9,69 & 0,380 \\
\hline Serrote & 14,8 & 16,3 & 0,475 \\
\hline Costela & $16,4^{\mathrm{a}}$ & $12,8^{\mathrm{b}}$ & 0,596 \\
\hline Pescoço & 8,16 & 8,87 & 0,255 \\
\hline
\end{tabular}

${ }^{\epsilon} \mathrm{F}_{1}$ SI x RL: Santa Inês x Rabo Largo; ${ }^{\complement} \mathrm{RL}$ : Rabo Largo; ${ }^{*} \mathrm{EPM}=$ Erro-padrão da média. Valores na mesma linha, seguidos de letras diferentes, diferem estatisticamente $(\mathrm{P}<0,05)$ pelo teste Tukey $(\mathrm{P}<0,05)$.

Resultados similares encontrados neste estudo foram reportados por Araújo Filho et al. (2010) que em estudo realizado com cordeiros Morada Nova, Santa Inês e mestiços $F_{1}$ Dorper x Santa Inês obtiveram valores médios de rendimento de corte nobres de $63,9 \%$.

\section{CONCLUSÃO}

A utilização da raça Santa Inês como base paterna demonstra atender aos padrões de características de carcaça de qualidade de cordeiros Rabo Largo em sistemas de produção de carne.

\section{REFERÊNCIAS}

AMARAL, R.M.; MACEDO, F.A.F.; ALCALDE, C.R. et al. Desempenho produtivo e econômico de cordeiros confinados abatidos com três espessuras de gordura. Rev. Bras. Saúde Prod. Anim., v.12, p.155$165,2011$.

ARAÚJO FILHO, J.T.; COSTA, R.G.; FRAGA, A.B. et al. Desempenho e composição da carcaça de cordeiros deslanados terminados em confinamento com diferentes dietas. Rev. Bras. Zootec., v.39, p.363$371,2010$.
BARROS, N.N.; VASCONCELOS, V.R.; WANDER, A.E.; ARAÚJO, M.R.A. Eficiência bioeconômica de cordeiro F1 Dorper x Santa Inês para produção de carne. Pesqui. Agropecu. Bras., v.40, p.825-831, 2005.

BRASIL. Decreto n. 30,691, alterado pelos Decretos n. 1,255 de 25- 06-62, n. 1236 de 02-09-94, n. 1.812 de 08-02-96 e n. 2.244 de 04-06-97. Aprova o regulamento da inspeção industrial e sanitária de produtos de origem animal (RIISPOA). Diário Oficial, Brasília, 5 jul. 1997. Seção I, p.11555.

CARNEIRO, P.L.S.; MALHADO, C.H.M.; SOUZA JUNIOR, A.A.O. et al. Desenvolvimento ponderal e diversidade fenotípica entre cruzamentos de ovinos Dorper com raças locais. Pesqui. Agropecu. Bras., v.42, p.991-998, 2007.

CARTAXO, F.Q.; SOUSA, W.H.; COSTA, R.G. et al. Características quantitativas da carcaça de cordeiros de diferentes genótipos submetidos a duas dietas. Rev. Bras. Zootec., v.40, p.2220-2227, 2011.

CARVALHO, J.A. Caracterização da atividade reprodutiva de fêmea ovina da raça Rabo Largo no semiárido do nordeste brasileiro. 58f. 2013. Tese (Doutorado em Zootecnia) - Universidade Estadual do Sudoeste da Bahia, Itapetinga. BA. 
CEZAR, M.F.; SOUSA, W.H. Carcaças ovinas $e$ caprinas: obtenção, avaliação e classificação. Uberaba: Agropecuária Tropical, 2007. 147p.

COSTA, R.G.; SANTOS, N.M.; SOUSA, W.H. et al. Qualidade física e sensorial da carne de cordeiros de três genótipos alimentados com rações formuladas com duas relações volumoso: concentrado. Rev. Bras. Zootec., v.40, p.1781-1787, 2011.

COSTA, R.L.D.; QUIRINO, C.R.; ALFONSO, V.A.C. et al. Morphometric indices in Santa Ines sheep. Int. J. Morphol., v.32, p.1370-1376, 2014.

CUNHA, E.A.; BUENO, M.S.; SANTOS, L.E. et al. Desempenho e características de carcaça de cordeiros Suffolk alimentados com diferentes volumosos. Ciênc. Rural, v.31, p.671-676, 2001.

FURUSHO-GARCIA， I.F.; COSTA， T.I.R.; ALMEIDA, A.K. et al. Performance and carcass characteristics of Santa Inês pure lambs and crosses with Dorper e Texel at different management systems. Rev. Bras. Zootec., v.39, p.1313-1321, 2010.

MADRUGA, M.S.; ARAÚJO, W.O.; SOUZA, W.H. et al. Efeito do genótipo e do sexo sobre a composição química e o perfil de ácidos graxos da carne de cordeiros. Rev. Bras. Zootec., v.35, p.1838-1844, 2006.

MARTINS, R.C.; OLIVEIRA, N.; OSORIO, J.C.S.; OSORIO, M.T.M. Peso vivo ao abate como indicador do peso e das características quantitativas $e$ qualitativas das carcaças em ovinos jovens da raça Ideal. Bagé: Embrapa Pecuária Sul, 2000. 29p. (Boletim de Pesquisa, 21).

MARTINS, S.R.; CHIZZOTTI, M.L.; YAMAMOTO, S.M. et al. Carcass and non-carcass component yields of crossbred Boer and Brazilian semiarid indigenous goats subjected to different feeding levels. Trop. Anim. Health Prod., v.46, p.647-653, 2014.

McMANUS, C.; PAIVA, S.R.; MELO, C.B.; SEIXAS, L. Raça ovina Rabo Largo. Brasília: Universidade de Brasília, 2010. 5p. (Série Técnica).

NUTRIENT of small ruminants: sheep, goats, cervids, and new world camelids. Washington: National Academy Press, 2007. 384p.

OFFICIAL methods of analysis. 18.ed. Gaithersburg, Maryland: AOAC, 1990.

OLIVEIRA, D.S.; ROGÉRIO, M.C.P.; BATISTA, A.S.M. et al. Desempenho e características de carcaça de cordeiros SPRD cruzados com as raças Santa Inês e Somalis Brasileira terminados em confinamento. Rev. Bras. Saúde Prod. Anim., v.15, p.937-946, 2014.
OSÓRIO, J.C.S.; OLIVEIRA, N.M.; OSÓRIO, M.T.M. et al. Produção de carne em cordeiros Cruza Border Leicester com ovelhas Corriedale e Ideal. Rev. Bras. Zootec., v.31, Supl., p.1469- 1480, 2002.

OSÓRIO, J.C.S.; OSÓRIO, M.T.M.; JARDIM, P.O.C. et al. Métodos para avaliação da produção da carne ovina: in vivo, na carcaça e na carne. Pelotas: UFPEL, 1998. p.107.

PIOLA JÚNIOR, W.; RIBEIRO, E.L.A.; MIZUBUTI, I.Y. et al. Ganho de peso e características da carcaça de cordeiros recebendo diferentes níveis de energia na ração. Semin. Ciênc. Agrár., v.30, p.935-944, 2009.

POMPEU, R.C.F.F.; CÂNDIDO, M.J.D.; PEREIRA, E.S. et al. Desempenho produtivo e características de carcaça de ovinos em confinamento alimentados com rações contendo torta de mamona destoxificada em substituição ao farelo de soja. Rev. Bras. Zootec., v.41, p.726-733, 2012.

SILVA SOBRINHO, A.G. Criação de ovinos. Jaboticabal: FUNEP, 2001. 302p.

SILVA SOBRINHO, A.G.; PURCHAS, R.W.; KADIM, I.T.; YAMAMOTO, S.M. Características de qualidade da carne de ovinos de diferentes genótipos e idades ao abate. Rev. Bras. Zootec., v.34, p.1070$1078,2005$.

SILVA, N.V.; SILVA, J.H.V.; COELHO, M.S. et al. Características de carcaça e carne ovina: uma abordagem das variáveis metodológicas e fatores de influência. Acta Vet. Bras., v.2, p103-110, 2008.

SIQUEIRA, E.R.; SIMÕES, C.D.; FERNANDES, S. Efeito do sexo e do peso ao abate sobre a produção de carne de cordeiro. Morfometria da carcaça, pesos dos cortes, composição tecidual e componentes não constituintes da carcaça. Rev. Bras. Zootec., v.30, p.1299-1307, 2001

STATISTICAL analysis system. Version 9.0. Cary: SAS Institute, 2001.

TILLEY, J.M.A.; TERRY, R.A. A two-stage technique for the in vitro digestion of forage crops. $J$. Br. Grassl. Soc., v.18, p.104-111, 1963.

VAN SOEST, P.J.; ROBERTSON, J.B.; LEWIS, B.A. Methods for dietary fiber, neutral detergent fiber, and nonstarch polysaccharides in relation to animal nutrition. J. Dairy Sci., v.74, p.3583-3597, 1991. 